\title{
AN ADVANCED WORKFLOW FOR SIMULATING HIGH RESOLUTION WIND FIELDS OVER COMPLEX URBAN TERRAIN INCLUDING SINGLE TREE OBJECTS
}

\author{
Maximilian Langheinrich ${ }^{1}$, Katja Seifert ${ }^{2}$ \\ ${ }^{1}$ German Aerospace Center, Remote Sensing Technology Institute, Weßling, Germany \\ ${ }^{2}$ FHWS, Faculty of Plastics Engineering and Surveying,Würzburg, Germany
}

Index Terms - OpenData, OpenFOAM, CFD, Simulation, Windspeed, Urban, City Model, Complex Terrain, OpenStreetMap, Remote Sensing, Geo Data

\section{INTRODUCTION}

Regarding urban environments, atmospheric models are often used to simulate processes, like i.e. heat island formation or the transportation of trace gases and particles, which are strongly connected to health and quality of living issues in modern cities. In the course of simulating atmospheric processes, wind speed and direction are often considered an important parameter. While freely available meteorological data provided by the national German Weather Service (DWD), as well as sparse in situ measurements are often used to feed atmospheric models of this kind, the low resolution (i.e. $1 \mathrm{~km}$ grid-size for DWD raster data) is not sufficient to represent small scale wind effects. Especially in urban environments effects like the amplification of wind in urban canyons acting as wind channels have a strong impact on how harmful air components spread. In order to model the transport of trace gases and particles in an urban environment with sufficient accuracy, higher resolution wind and pressure data is necessary.

This paper presents a novel approach for the generation of high-resolution wind speed and atmospheric pressure field data on the basis of freely available geo data from different sources and the OpenFoam open-source computational fluid dynamics (CFD) framework which subsequently can be used as input parameters to improve the accuracy of atmospheric models.

\section{METHODOLOGY}

\subsection{Data acquisition approaches}

Two different data sources were implemented to act as the basic input for the model preparation. Recently several German federal surveying offices introduced new open data policies providing a large number of freely available and highly accurate geo data to the public. These include Digital Elevation Models (DEMs), Digital Terrain Models (DTMs) and LOD2 Building Models which can be utilized to generate a very descriptive representation of a complex urban terrain.

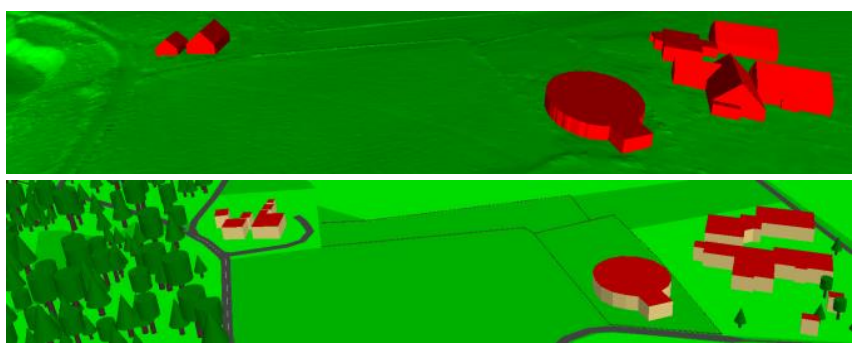

Figure 1. Extracted and meshed LOD2/DTM based (top) and OSM based (bottom) 3D models.

OpenStreetMap (OSM) datasets on the other hand feature a multitude of different information, including street layout and different types of single vegetation objects. The accuracy of the geo data in OpenStreetMap datasets is not guaranteed to be as accurate and complete as the data from official sources. Especially the existence of single tree object information is of particular interest concerning the described process.

Regarding the two different possibilities of acquiring elementary 3D urban geometry, individual methods were applied to extract the data. In order to generate a complex urban terrain model from federal surveying data, a processing chain was developed in Python that parses the geo database for LOD2 and DTM data for a particular test area, fuses the point data into one dataset and meshes the point cloud into a coherent surface.

OSM data is processed by a Python wrapper utilizing the OSM2World software developed at the University of Delft [5]. In both cases the surface models are stored and further processed in the Wavefront $O B J$ format. Figure 1 shows example representation of the resulting 3D models.

\subsection{Atmospheric volume meshing}

Defining an atmospheric volume around the complex urban terrain and meshing it into individual cells represents a critical preprocessing step of the computational fluid 
dynamics simulation, as the latter solves the Navier-Stokes equations of turbulent flow by means of finite element calculations. While there exist several commercial and open source solutions for preparing volumetric meshes (i.e. as shown in [7]), the best results can be achieved by using the snappyHexMesh meshing software included in the OpenFOAM framework itself [9].

\subsection{Modeling trees}

One of the most challenging tasks in former experiments concerning the simulation of wind fields over complex urban terrain was the implementation of the effect of vegetation on the turbulent propagation of air. Vegetation areas were simulated by dividing the DTM surface into different physical boundary zones on the basis of landuse classification datasets [8] and applying a roughness length parameter to the individual zones. This approach has turned out as an insufficient representation of vegetation impact, as the roughness length is more of a meteorological macroscopic parameter not meeting the requirements for a small scale high resolution representation as generated in the proposed approach.
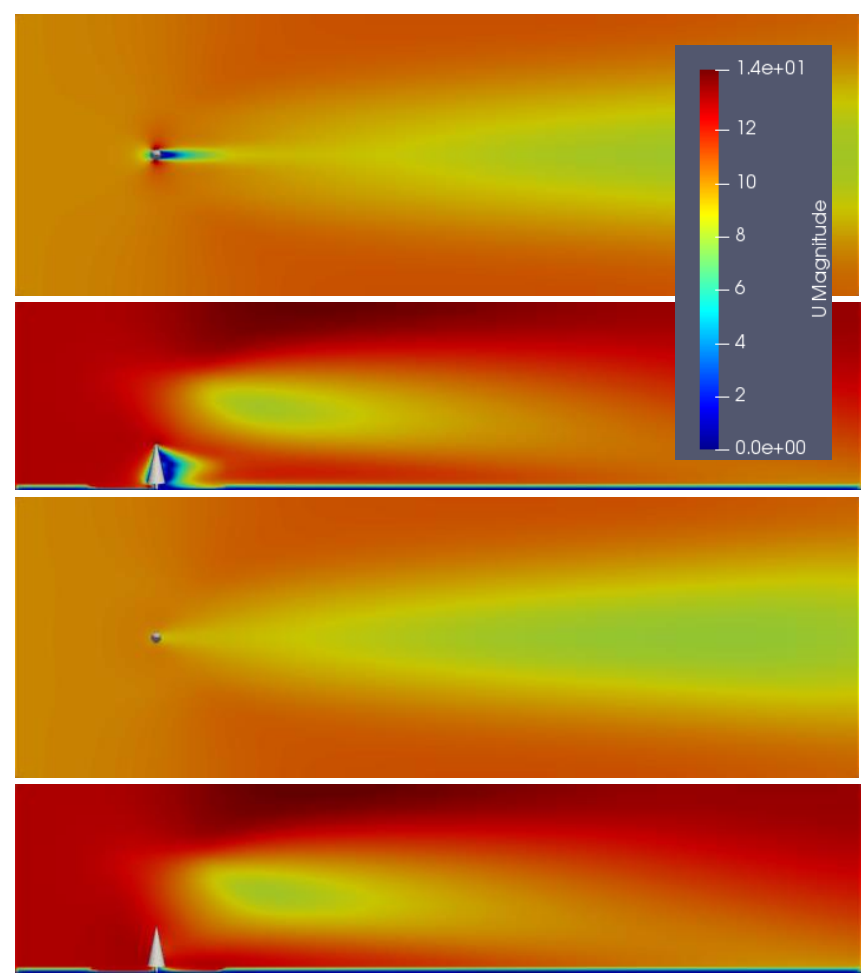

Figure 2. Top and side views of single tree simulation wirh fully blocking tree ( 2 top) and porous tree ( 2 bottom).

As it is considered that vegetation featured within an urban environment has a strong influence on how wind propagates, alternative approaches had to be developed to implement a sufficient representation.

With the possibility of extracting individual tree objects from OSM data, two different solutions have been tested. The more simple approach is to model the individual trees as impenetrable objects (like i.e. building objects). While this already resembles are more improved representation of high resolution vegetation, it does not describe the real behavior of trees concerning wind.

The latest OpenFOAM version enables the user to define cells of porous media which do not block the propagation of the turbulent flow of air through these particular cells to the full extent. The porosity is implemented with the Darcy-Forchheimer-Equations for turbulent flow through porous media [12] for the affected vegetation cells. Figure 2 depicts the different behavior of turbulent air flows concerning fully blocking and porous modeling of tree objects.

\subsection{CFD simulation}

For the computational fluid dynamics calculations a simplified version of the Navier-Stokes equations of turbulent flow is used on basis of the following postulations:

- The air, affected by the turbulent flow, can be treated as an incompressible fluid in the on hand simulation as the simulated wind speed is at $10 \mathrm{~m} / \mathrm{s}$ which adds up to a low Mach-Number of 0.029 [11].

- A constant value is considered for the boundary conditions of wind speed and air pressure resulting in a steady-state realization of the equations.

The steady state approach converges to a stable solution of the Navier-Stokes calculations that can be seen as a mean distribution of wind speed and pressure values over the extents of the atmospheric boundary volume.

Further a standard RANS (Reynolds-averaged NavierStokes) turbulence model is chosen in form of the Lam and Bremhorst low-Reynolds number k- $\varepsilon$ turbulence model [6].

\section{DATA AND EXPERIMENTS}

The underlying 3D model was extracted from OpenStreetMap data using OSM2World in order to allow the modeling of individual vegetation objects. As at the current state no method was implemented to fuse the OSM extracted model with DTM data, the earth surface is modeled as a flat plane approximation at height 0 meters.

The area of interest chosen for the on hand experiments is located near the center of Munich, Germany. It covers a region extending from the upper left corner with longitude $11.53^{\circ}$, latitude $48.15^{\circ}$ to the lower right corner with longitude $11.54^{\circ}$ and latitude $48.14^{\circ}$ comprising an area of 
636 meters in $\mathrm{x}$-direction and 1022 meters in y-direction respectively. The highest point of the urban terrain has a build-up height of 54 meters.

It is common practice to increase the dimensions of the atmospheric volume beyond the extents of the observed test area to provide sufficient space for the turbulent flow to develop. This was conducted according to the following criteria:

- The blocking ratio not exceeding 3\%, where the blocking ratio is the ratio between building vertical surfaces exposed to the wind and the surface of the simulation inlet $[1][10]$.

- The minimum dimensions of the simulated field is $5 * \mathrm{H}$ (with $\mathrm{H}$ as the height of the highest building) upstream of the buildings, $5 * \mathrm{H}$ on each side of the buildings, $5 * \mathrm{H}$ above the buildings and $15 * \mathrm{H}$ downstream [4][10].

By rounding the new dimensions to exponentials of 2 to ensure the correct automatic refinement of the initial cells down to a resolution of 1 meter side length, the final extents of the atmospheric volume are 2048 meters x 2048 meters x 512 meters.

The initial cell size of the atmospheric volume mesh was set to 16 meters with a refinement factor of 3 around building objects, where one refinement step is defined as a division of the cell side length by 2 , resulting in a final resolution of 2 meter.

Concerning the porosity configuration of the DarcyForchheimer model the coefficients $d$ and $f$ were set to $200 / \mathrm{m}^{2}$ and $0 / \mathrm{m}$ respectively.

The initial boundary conditions of the CFD calculation are set to $10 \mathrm{~m} / \mathrm{s}$ for wind speed, with the wind coming from true west direction, and an atmospheric pressure of 1013.25 $\mathrm{hPa}$. The simulation is iterated over a period of 400 seconds with an iteration step size of 1 second.

\section{RESULTS}

Figure 2 shows the overall, error-free convergence of the CFD calculation process with final residuals for the model parameters of $8.12 \mathrm{e}-7 \mathrm{~m} / \mathrm{s}, 2.57 \mathrm{e}-5 \mathrm{~m} / \mathrm{s}, 1.48 \mathrm{e}-5 \mathrm{~m} / \mathrm{s}$ for the windspeed in X-, Y- and Z-dimension (Ux, Uy, Uz), $1.34 \mathrm{e}-4 \mathrm{hPa}$ for atmospheric pressure(p), 9.88e-6 for $\varepsilon$ and $1.23 \mathrm{e}-5$ for $\mathrm{k}$ (both dimensionless). This shows that the underlying meshing process on basis of the OSM data produces a mesh of sufficient quality, resulting in a very stable steady-state solution.

Figure 3 exemplary visualizes the wind field plane for the steady-state wind speed values for cells 2 meters above the flat plane ground.

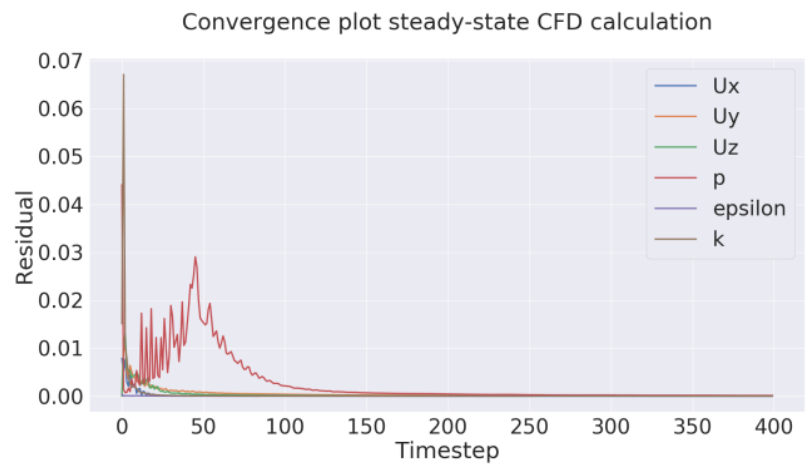

Figure 2. Convergence plot of CFD calculations.

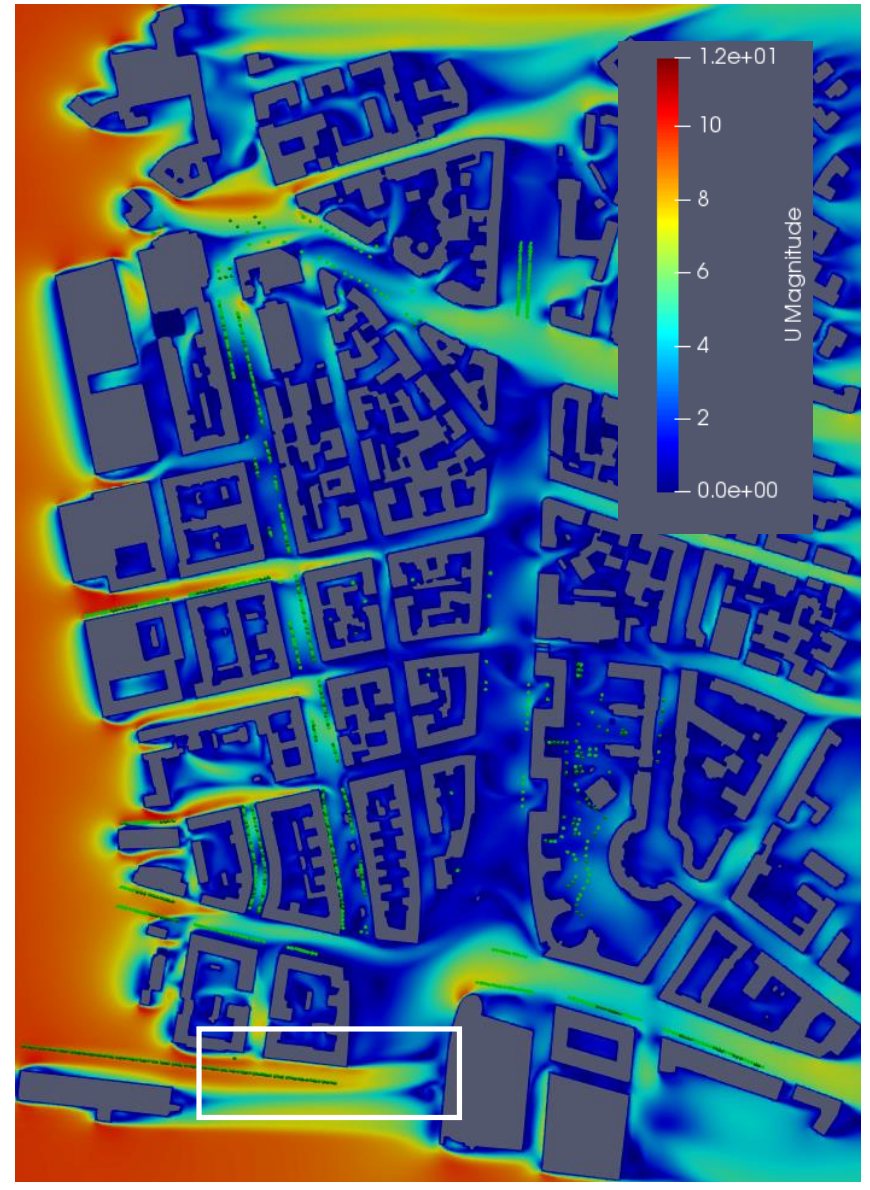

Figure 3. Steady-state wind speeds over complex urban terrain 2 meters above ground.

The microscale effects of wind tunnel amplifications in street canyons can clearly be seen in the direction of the wind flow. While most of the momentum is slowed down by the west facing building facades and partly by porous vegetation. The latter is shown in the close up comparison in Figure 4 where it can clearly seen how windspeeds decrease by passing porous trees. Stronger currents propagate into the streets in parallel direction to the attack direction. Further 
the formation of eddies can be observed in the area of the large street in the middle of the test area (north-south direction).

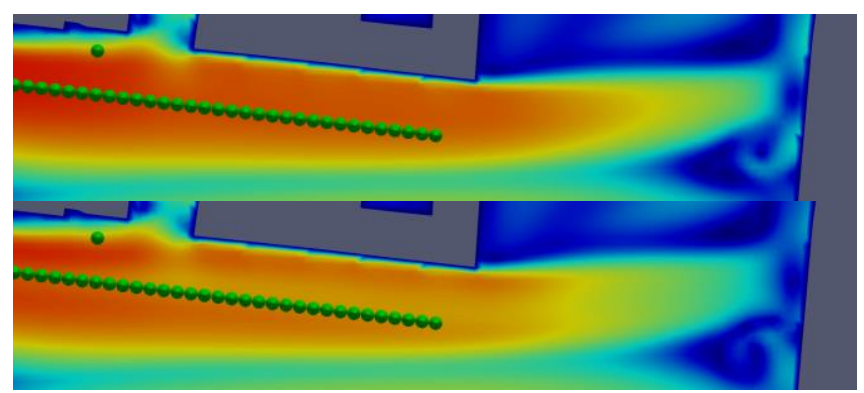

Figure 4. Close up of marked area (Figure 3). Vegetation not taken into account (top) and with porous modeling (bottom).

This observation is of particular importance as this street is a main traffic area which is considered one of the main sources for inner city fine particulate air pollution. All of these described and observed effects can only be made visible by high-resolution models as proposed in this work.

The simulated dataset consists of 5895946 data points and 9197040 data cells, all featuring values for Ux, Uy, Uz and the overall magnitude of $\mathrm{U}$, as well as air pressure values. Therefore it delivers an extensive data basis for the implication into atmospheric models.

\section{CONCLUSION}

In this paper a workflow was presented to generate high-resolution wind speed and atmospheric pressure datasets from publicly available geo data sources. The produced results can be judged as highly satisfying with a high potential to increase the accuracy of models describing atmospheric transportation processes.

While it was possible to improve preceding work [7][8] concerning individual cell size, overall test area extents and the realistic modeling of vegetation objects, there is still space for further advancements. Although the implementation of individual vegetation objects as porous media cells is seen the most realistic approach achieved up to now compared to past experiments, it still has to be evaluated which porosity coefficient values best approximate generalized vegetation.

Further a solution to generate homogeneous datasets from the two present data sources, either by fusing the OSM datasets with a digital terrain model, or by introducing individual tree objects to the fused LOD2 / DTM model still has to be developed. The latter approach is considered more promising as the data provided by federal surveying offices exhibit a much higher accuracy compared to OpenStreetMaps, while also providing important building features like roof types which are arbitrarily in OSM.

\section{REFERENCES}

[1] Baetke, F., Werner, H., \& Wengle, H., "Numerical simulation of turbulent flow over surface-mounted obstacles with sharp edges and corners", Journal of Wind Engineering and Industrial Aerodynamics, 35, 129-147. 1990.

[2] Bitog, J. P., et al. "A wind tunnel study on aerodynamic porosity and windbreak drag." Forest Science and technology 7.1, pp. 8-16, 2011.

[3] Cao, Jinxin, Yukio Tamura, and Akihito Yoshida. "Wind tunnel study on aerodynamic characteristics of shrubby specimens of three tree species." Urban forestry \& urban greening 11.4, pp. 465-476, 2012.

[4] Hall, R. C., "Evaluation of modelling uncertainty. CFD modelling of near-field atmospheric dispersion. Project EMU final report", European Commission Directorate-General XII Science. Research and Development Contract EV5V-CT94-0531, WS Atkins Consultants Ltd., Surrey. 1997.

[5] Knerr, T., "OSM2World Create $3 D$ models from OpenStreetMap.”, [Online]. Available: http://osm2world.org/, Accessed: 02.01.2019.

[6] Lam, C. K. G., \& Bremhorst, K., "A modified form of the $k-\varepsilon$ model for predicting wall turbulence.", Journal of fluids engineering, 103(3), pp. 456-460. 1981.

[7] Langheinrich, M. "Evaluation of gmsh meshing algorithms in preparation of high-resolution wind speed simulations in urban areas.", International Archives of the Photogrammetry, Remote Sensing \& Spatial Information Sciences 42.2, 2018.

[8] Langheinrich, M., Fischer, P., \& Krauß, T., "Modeling wind flow over complex urban terrain". In Urban Remote Sensing Event (JURSE), 2017 Joint (pp. 1-4). IEEE. 2017.

[9] "OpenFOAM v6 User Guide: 5.4 Mesh generation with snappyHexMesh.", [Online], Available: http://cfd.direct/openfoam/user-guide/v6-snappyhexmesh/,

Accessed: 04.01.2019.

[10] Reiter, Sigrid., "Validation process for CFD simulations of wind around buildings." Proceedings of the European Built Environment CAE Conference. Londres, 2008.

[11] Tritton, D. J., Physical fluid dynamics, Springer Science \& Business Media. 2012.

[12] Whitaker, S., "The Forchheimer equation: a theoretical development.", Transport in Porous media 25.1, pp. 27-61, 1996. 